\title{
Electron Matter-Waves as Electromagnetically Induced Crystal Oscillator Effects
}

Joseph A Hazani ( $\square$ joseph@adilettante.com )

\section{Research Article}

Keywords: Matter-Waves, Davisson-Germer Experiment, Bragg's Law, X-Ray Crystallography, Crystal Oscillator

Posted Date: April 8th, 2021

DOl: https://doi.org/10.21203/rs.3.rs-403344/v1

License: (c) (i) This work is licensed under a Creative Commons Attribution 4.0 International License. Read Full License 


\section{Electron Matter-Waves as Electromagnetically Induced Crystal Oscillator Effects}

I. Introduction

The famed Davisson-Germer Experiments demonstrated the wave phenomenon of electrons similarly to X-Ray scattering from Sir Lawrence Bragg's X-ray experimentations on crystals c. 1913. Their empirical deduction of electrons behaving as waves (i.e. oscillatory) ignores the possibility of an electron beam behaving harmonically upon elastic collision with a diffraction grating represented by nickel crystal - in their experiment. However, it is well established in the electrical engineering science that crystals possess piezoelectric effects and are used ubiquitously in electronic circuit designs for causing stable harmonic oscillation responses to direct current voltages. In light of this, the current mathematical model proposes the Davisson-Germer results to be the effect of a nickel crystal oscillator circuit which amplifies a direct voltage source - the electron beam - causing the phenomenon of inductance from the resultant electrical feedback with the crystal atom's electromagnetic field.

Clinton Davisson and Lester Germer conducted a series of famous experiments in the 1920's which provided ammunition to an unresolved quandary: what is the physical nature of waveparticle duality? Their observation of electron beam reflections which amplify intensities, in similarity with X-Ray crystal reflection experiments by William and Sir Lawrence Bragg, provided the foundation for their theoretical investigation of the so called "matter-wave" hypothesized by Alan de Broglie. Yet the history of the determination of the electron wavelength experimental quantity, which awarded de Broglie and Davisson the Nobel Prize in 1929 and 1937, respectively, requires an understanding of the advancements in crystallography physics which the Experimenters found congruency with. Namely, the X-ray crystallography experiments from the father and son Bragg team.

\section{Crystal Lattice Structure}


The Bragg's experimental history was inspired by the newfound discovery of X-rays and the conceivability of empirical phenomena on the quantum scale. Bragg et al determined the atomic spacing of their experimental crystals effecting "special reflection angles" of "homogenous x-rays":

"It often happens that the rays emerging from the bulb slit and falling on the crystal contain a large preponderance of rays of a given quality which can only be reflected at a certain angle... When... we see a maximum persisting in the same angular position...for several successive positions of the crystal, we know that we have a case of this special reflection. There is a relatively large quantity of very homogeneous radiation of a certain kind present in the radiation from the bulb."”2 (emphasis added)

Bragg proposed an explanation for this homogeneity by conceiving of the crystal as a lattice of atoms:

"There is strong evidence for supposing that the atoms of a cubic crystal-like rock-salt, containing two elements of equal valency, are arranged parallel to the planes $\{100\}$ in planes containing equal numbers of sodium and chlorine atoms. The atoms in any one plane are arranged in alternate rows of each element, diagonal to the cube axes, successive planes having these rows opposite ways."3

Yet their static geometrical optics relationship (1), diagraming what has been determined to be an elastic collision phenomenon, does not sufficiently describe the dynamics of energy transfer in the observable system. In fact, it is admitted that the "special reflections" are distinct between crystals:

"The angles at which the special reflections of these rays take place are not the same for all crystals, nor for all faces of the same crystal."

\footnotetext{
${ }^{1}$ Bragg William Henry and Bragg William Lawrence 1913. The reflection of X-rays by crystals. Proc. R. Soc. Lond. A88428-438. http://doi.org/10.1098/rspa.1913.0040

${ }^{2}$ Ibid.

${ }^{3}$ Ibid.

${ }^{4}$ Ibid.
} 


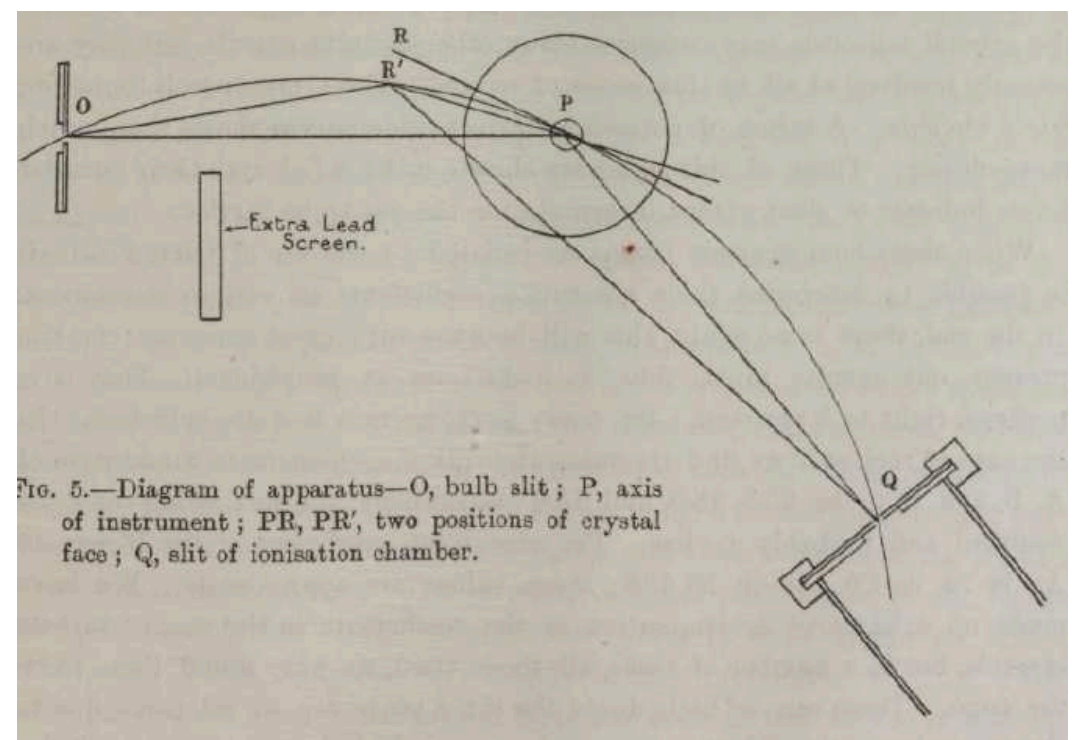

Figure A Bragg Experimental Setup with X-ray emitting from a point source $O$ and reflecting at a "special angle".

Furthermore, it is important for modeling considerations to mention Bragg's observation's pertinence to wave-particle duality, despite the conclusive diffraction effect witnessed:

"These results do not really affect the use of the corpuscular theory of X-rays. The theory represents the facts of the transfer of energy from electron to X-ray and vice versa, and all the phenomena in which this transfer is the principal event."

Yet despite these comments of their results, that the X-ray scattering can still theoretically be conceived of as a cause of a corpuscular energy transfer and therefore possible model "classically",

The Braggs did not believe the homogeneous rays were being "manufactured in the crystal":

"There can be little doubt as to the interpretation of these results... Rays of a definite quality are reflected from a crystal when, and only when, the crystal is set at the right angle. This is really an alternative way of stating the original deduction of [Max von] Laue. The three sets of rays are not manufactured in the crystal, because all their properties are independent of the nature of the crystal."

But what is meant by "manufactured”? In referencing Max von Laue, Bragg's experiment confirmed the X-ray diffractions of the 1920 Nobel Prize Winner which, at his address, states:

\footnotetext{
${ }^{5}$ Ibid.

${ }^{6}$ Ibid.

${ }^{7}$ Ibid.
} 
"It was left to William Lawrence Bragg to demonstrate that the diffraction pattern was due to the reflection of the 'white' Bremsstrahlen of the primary beam on the crystal planes that selected certain wavelengths for the diffraction pattern by what became known as the 'Bragg condition'." 8

In other words, the question of whether the X-rays were an effect, i.e. "manufactured", of

electromagnetic energy released from the lattice electrons during the X-ray beam collision was

answered: the crystal was acting like a diffraction grating for the primary monochromatic X-ray

beam, with a precise relation to its atomic lattice spacing. ' Laue’s own Nobel Address further

remarks on this curiosity: ${ }^{10}$

"I was suddenly struck by the obvious question of the behaviour of waves which are short by comparison with the lattice-constants of the space-lattice. And it was at that point that my intuition for optics suddenly gave me the answer: lattice spectra would have to ensue. The fact that the lattice-constant in crystals is of an order of $10^{-8} \mathrm{~cm}$ was sufficiently known from the analogy with other interatomic distances in solid and liquid substances, and, in addition, this could easily be argued from the density, molecular weight and the mass of the hydrogen atom which, just at that time, had been particularly well determined. The order of $\mathrm{X}$-ray wavelengths was estimated by Wien and Sommerfeld at $10^{-9} \mathrm{~cm}$. Thus, the ratio of wavelengths and lattice-constants was extremely favourable if X-rays were to be transmitted through a crystal. I immediately told [Paul Peter] Ewald that I anticipated the occurrence of interference phenomena with X-rays."

Yet, upon such a conclusion, there is no consideration of the natural harmonics of the "lattice-space hypothesis" medium of transmission in Braggs' nor Laue's analysis. Indeed, it is self-evident there

\footnotetext{
${ }^{8}$ Eckert, Michael. "Max Von Laue and the Discovery of X-Ray Diffraction in 1912.” Ann. Phys., vol. 5, 7 May 2012, pp. A83-A85., doi:10.1002/andp.201200724.

${ }^{9}$ Eckert, M., 2012. Disputed discovery: the beginnings of X-ray diffraction in crystals in 1912 and its repercussions. Acta Crystallographica, A68(1), 30-39.

${ }^{10}$ Laue, Max Von. “Concerning the Detection of X-Ray Interferences.” Nobel Address. 3 June 1920.
} 
are crystal lattice atomic movements which occur as vibrations (lattice vibrations). ${ }^{1112}$ And due to the regularity of the atoms, it is intuitive to hypothesize the uniform, i.e. isotropic, motion of atoms as a piezoelectric oscillator, as has been proposed. ${ }^{13}$

This intuition was apparent during the time of these experiments. The animation of the atoms by de facto thermal motion is what Peter Debye aimed to characterize in the X-ray crystallography experiments in $1913 .{ }^{1415}$ However, it must be mentioned that Debye's model assumes photon-electron energy transfer, and thus includes by necessity an elastically determined electrodynamical effect on the x-ray beam:

\begin{abstract}
"It was at this time that Mark and Wierl presented a preliminary description of their investigations showing that the Debye formula descriptive of the scattering of $\mathrm{x}$ rays by a gas...could also be applied to describe the scattering of electron rays by gases. Physically, there is one difference. The electron interferences provide information about the positions of the atomic nuclei themselves, while the x-ray interferences reveal the locations of the centers of gravity of the electron clouds about them. What is really ascertained in either case is the position of the atom centers, the desired quantity."
\end{abstract}

In other words, when including the "thermal fluctuations" within the crystal atom in interpreting the diffraction results, the X-ray (or electron ray) atomic collision in a lattice crystal can still be conceived of as classical elastic energy transfer. Yet still, a piezoelectric oscillator, i.e. resonator, model

\footnotetext{
11 Symbal, E.Y.T. "Section 5: Lattice Vibrations." Section 5: Lattice Vibrations. unlcms.unl.edu/cas/physics/tsymbal/teaching/SSP-927/Section\%2005_Lattice_Vibrations.pdf.

12 M Suzuki, IS Suzuki. "A proper understanding of the Davisson and Germer experiments for undergraduate modern physics course.”- arXiv preprint arXiv:1307.6049, 2013 - arxiv.org

13 “Lecture - 24 X-Ray Diffraction.” Performance by S. Bharadwaj, Lecture - 24 X-Ray Diffraction, IIT Kharagpur, 21 Jan. 2009, youtu.be/2CsMpEB15QY.

14 P. Debye, Ann. Phys. 1913, 43, 49-92.

15 Gelisio, Luca, and Paolo Scardi. "100 Years of Debye's Scattering Equation.” Acta Crystallographica, no. A72, 31 Oct. 2016, pp. 608-620., doi:10.1107/S2053273316014881.
}

16 Williams, J. (1975). Peter Josseph Wilhelm Debye (pp. 29-30). Washington, DC: National Academy of Sciences. doi:http://www.nasonline.org/publications/biographical-memoirs/memoir-pdfs/debye-peter.pdf 
of the crystal lattice initialized by an electromagnetic radiation disturbance (e.g. the X-ray beam) was not published by any of the researchers. Indeed, neither was there a consideration, assuming an isotropic crystal vibration, of the electric polarization effecting the motion of the electrons or x-rays. This, despite the advancements in the science of $\mathrm{x}$-ray diffraction which incorporates polarization corrections to the scattering angle, i.e. Lorentz-Polarization Correction, and the calculation of dipole effects on light scattering. ${ }^{1718}$

\section{De Broglie’s Matter-Wave Hypothesis}

Meanwhile, de Broglie's intuition was predicated on his curiosity of Max Plank's resolution of the UV catastrophe in black body radiators with the introduction of the "quantum" phenomenon:

"Planck was led to assume that only certain preferred motions, quantized motions, are possible or at least stable, since energy can only assume values forming a discontinuous sequence. This concept seemed rather strange at first but its value had to be recognized because it was this concept which brought Planck to the correct law of black-body radiation and because it then proved its fruitfulness in many other fields." ${ }^{\prime 19}$

He then investigated a relativistic relation between the wavelength of an electromagnetic wave and linear momentum out of necessity of taking into account the frequency nature of electromagnetic phenomena:

When I started to ponder these difficulties two things struck me in the main. Firstly the light-quantum theory cannot be regarded as satisfactory since it defines the energy of a light corpuscle by the relation $W=b v$ which contains a frequency $v$. Now a purely corpuscular theory does not contain any element permitting the definition of a frequency. This reason alone renders it necessary in the case of light to introduce simultaneously the corpuscle concept and the concept of periodicity. I thus arrived at the following overall concept which guided my studies: for both matter and radiations, light in particular, it is

17

https://chem.libretexts.org/Bookshelves/Inorganic_Chemistry/Modules_and_Websites_(Inorganic_Chemistry)/Crysta llography/X-rays/Lorentz-polarization_correction

18 ECE 695S Lecture 04: Light Interaction with Small Structures

(https://nanohub.org/resources/19363/supportingdocs)

${ }^{19}$ Broglie, Louis de. “The Wave Nature of the Electron.” Nobel Lecture. 12 Dec. 1929. 
necessary to introduce the corpuscle concept and the wave concept at the same time. In other words the existence of corpuscles accompanied by waves has to be assumed in all cases. ${ }^{20}$ (emphasis added)

It is this consideration which helped him derive a rectilinear measurement of periodical motion from theorizing the linear momentum of a light-quantum to move with a longitudinal phase velocity:

"The quantity; $\lambda$ is the distance between two consecutive peaks of the wave, i.e. the 'wavelength'. Hence:

$$
\lambda=\mathrm{h} \varrho
$$

This is a fundamental relation of the theory." ${ }^{21}$

This is the wave-momentum relation mathematically induced by de Broglie that corresponded to the scattering experienced and published by Davisson and Germer in their experiments on electron beams. In that, the Bragg relation of $x$-ray wavelength and crystal atomic spacing was determinant according to the similar geometric scattering angle of the x-ray energy in Bragg's crystal experiment:

$$
\lambda=2 \mathrm{~d} \sin \theta(1)
$$

Thus, Davisson and Germer analytically arrived at the hypothetical electron wavelength within de Broglie's relation when computing Bragg's relation - itself a priori assuming the geometrical optics of an X-ray wave reflectance - with the experimentally measured scattering angle of the electron. This was computed with a classical kinetic energy relation when applied to electrodynamical voltages:

$$
\lambda=\mathrm{h} / \sqrt{ }(2 \mathrm{mE})(2)
$$

This experiment, which presupposes the electron diffraction approximation between Bragg's scattering angle determination and de Broglie's a priori wavelength derivation, resulted in two Nobel

${ }^{20}$ Ibid.

${ }^{21}$ Ibid. 
prizes in 10 years: one for the theoretician and one for the experimentalist. From Davisson's Nobel

Address:

"It was brought out that light imparts energy to individual electrons in amounts proportional to its frequency and finally that the factor of proportionality between energy and frequency is just that previously deduced by Planck from the black-body spectrum. The idea of pressing the witness on the latter point had come from Einstein who outplancked Planck in not only accepting quantization, but in conceiving of light quanta as actual small packets or particles of energy transferable to single electrons in toto."22

And this was further confirmed by Compton:

The case for a corpuscular aspect of light, now exceedingly strong, became overwhelmingly so when in 1922 A. H. Compton showed that in certain circumstances light quanta photons as they were now called - have elastic collisions with electrons in accordance with the simple laws of particle dynamics. ${ }^{23}$

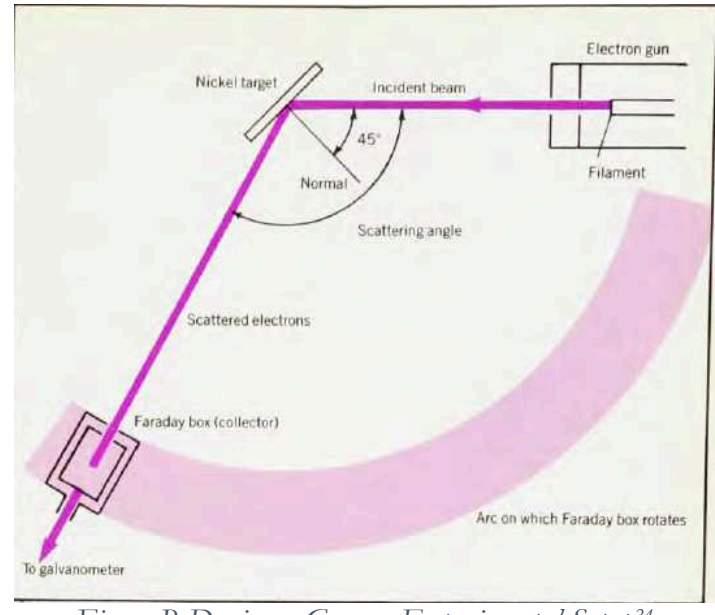

Figure B Davisson-Germer Experimental Setup ${ }^{24}$
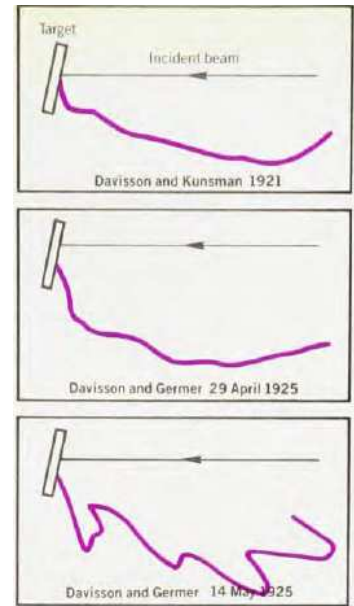

Figure C Electron Diffraction Experimental Results $5^{25}$

${ }^{22}$ Davisson, Clinton J. “The Discovery of Electron Waves.” Nobel Lecture. 13 Dec. 1937.

${ }^{23}$ Ibid.

${ }^{24}$ Gehrenbeck, Richard K. Electron Diffraction: Fifty Years Ago. Physics Today, January 1978. p37.

${ }^{25}$ Ibid p39. 
The experiments furthermore coincided with other discoveries of the wave motion behavior of matter, such as with helium and neutrons. ${ }^{26}$ Yet despite the matriculation of the concept of "matterwaves" from these series of experiments, there is a lack of discourse on the proper mathematical characterization of the motion, i.e. mechanics, in the observation system. Namely, Bragg's relation in equation (1) is determined from a theoretically stationary geometric optical model of crystal lattices and does not quantify the natural harmonic effects of the crystal's lattice vibrations upon the electromagnetic radiation scattering. And while such vibrations had been thoroughly investigated by Peter Debye, ${ }^{27}$ and later formulated into the Debye-Waller Factor, they do not mechanically diagram the crystal lattice's electromagnetic effects. Specifically, there is no discussion on the possibility of the diffraction results of the Davisson-Germer experiments as an effect of piezoelectric polarization.

\section{Piezoelectric Effects}

To more richly diagram the mechanics of the system, and to support the hypothesis that DavissonGermer's experimental results are the effects of electromagnetic induction, the consideration of voltage effects needs to be included, which would intuitively lead to electromechanical force diagramming. For the most plausible mathematical model of the crystal lattices mechanism of simple harmonic motion which amplifies electromagnetic reflections as an inductive effect of elastic energy

\footnotetext{
${ }^{26}$ Smith, Kevin. Matter Waves. Youtube / Matter Waves, Educational Services, Inc., 20 Jan. 2015, www.youtube.com/watch?v=szGJnpNowqw.

${ }^{27}$ Eckert, Michael. "Disputed Discovery: The Beginnings of X-Ray Diffraction in Crystals in 1912 and Its Repercussions." Acta Crystallographica Section A, vol. 68, ser. 1, Jan. 2012, pp. 30-39. 1, https://doi.org/10.1107/S0108767311039985.
} 
transfer collisions, an electromechanical, i.e. piezoelectric, phenomenon must be hypothesized. And indeed, the dipole moments in the crystal structure had been considered by Debye:

\begin{abstract}
"It is as a consequence of their asymmetrical (electrical) structure that most molecules possess a permanent dipole moment; the magnitude of this characteristic entity is a quantitative measure of the polarity of the molecule... Debye reasoned that such asymmetric molecules must possess finite and permanent electrical moments and that their total electrical polarizations result from two contributions, a displacement of electrons and atoms in the molecule and an orientation in the electrical field of the molecule as a whole." 28
\end{abstract}

The proposed mathematical model therefore hypothesizes an electromagnetic phenomenon in the collision - the electron electric field - causes a dielectric material effect, i.e. polarization, in the crystal, which is consistent with the Lorentz Harmonic Oscillator classical mechanical model of the harmonic effects of an electric field acting on an atom during an elastic collision. ${ }^{29}$
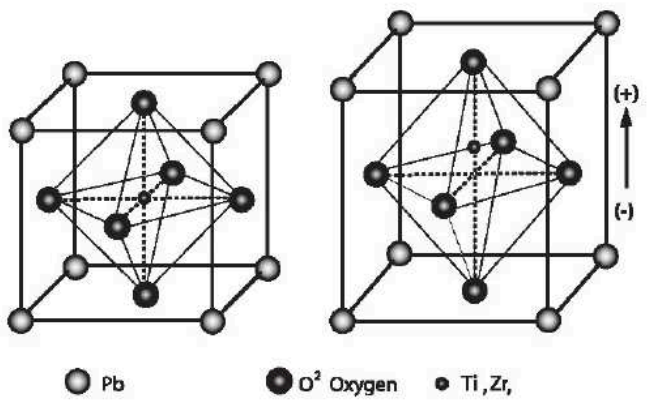

Figure 2.1. Crystalline structure of a piezoelectric ceramic, before and after polarization

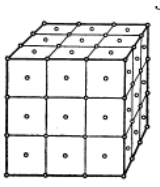

(a)

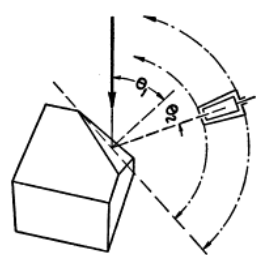

(c)

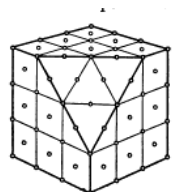

(b)

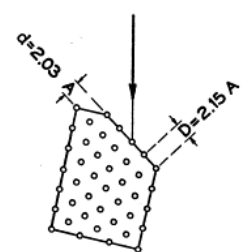

(d)

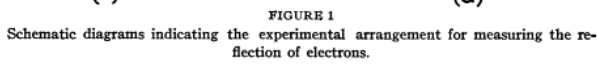

the experimental ans
flection of electrons.

\footnotetext{
${ }^{28}$ Williams, J. (1975). Peter Josseph Wilhelm Debye (p. 32). Washington, DC: National Academy of Sciences. doi:http://www.nasonline.org/publications/biographical-memoirs/memoir-pdfs/debye-peter.pdf

${ }^{29}$ Insert reference for Lorentz harmonic oscillator

${ }^{30}$ (2006) Fundamentals of Piezoelectricity. In: Piezoelectric Transducers for Vibration Control and Damping. Advances in Industrial Control. Springer, London. https://doi.org/10.1007/1-84628-332-9_2

${ }^{31}$ Davisson CJ, Germer LH. Reflection of Electrons by a Crystal of Nickel. Proc Natl Acad Sci U S A. 1928;14(4):317-322. doi:10.1073/pnas.14.4.317
} 


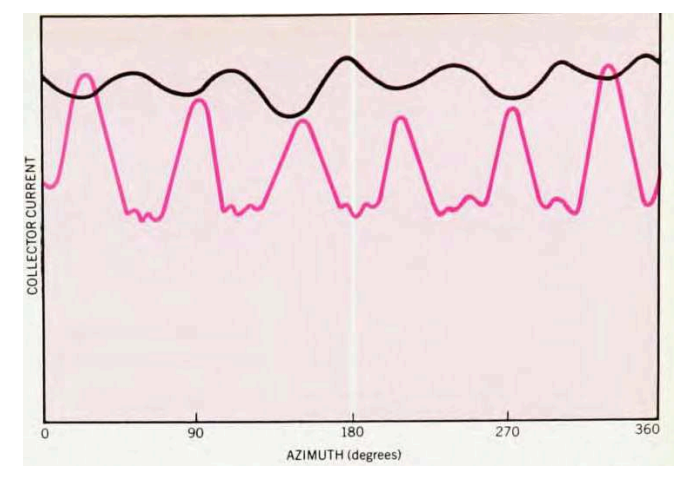

Figure F Davisson-Germer Scattering Results Suggesting Amplifier Gains of Piezoelectric Oscillation Effects ${ }^{2}$

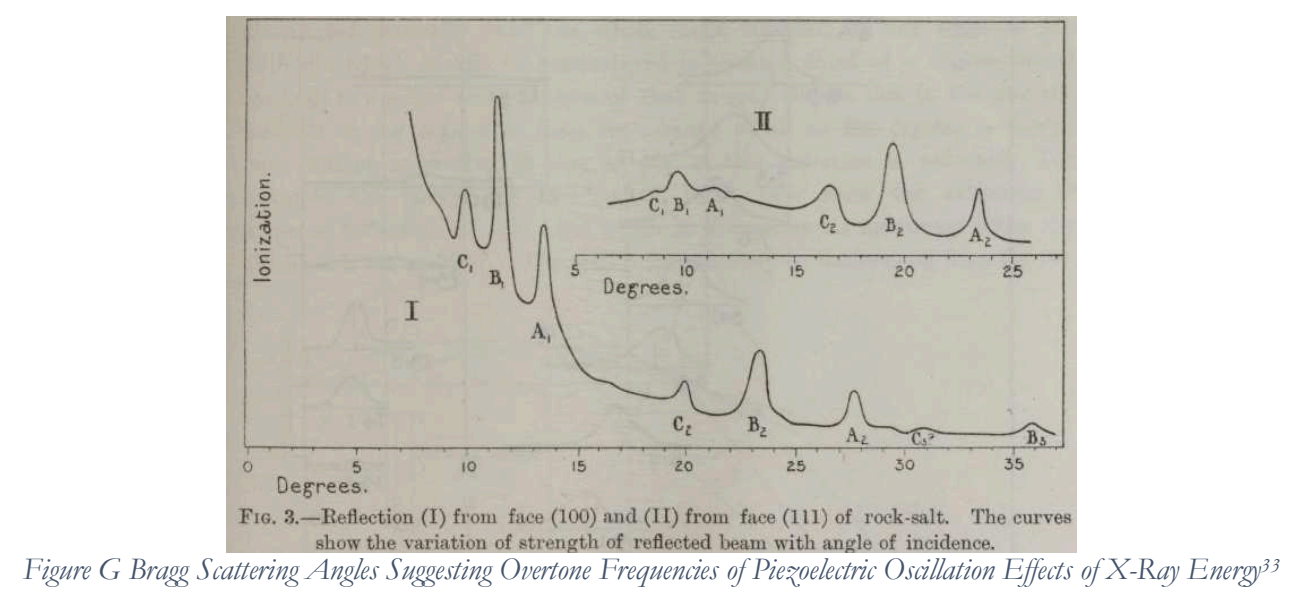

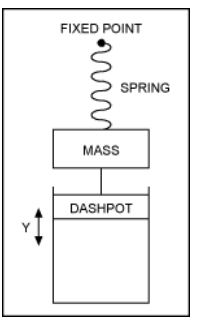

Figure H Damped Harmonic Oscillator Mechanical Model
${ }^{34}$ More precisely, the crystal lattice can hypothetically be mechanically modeled as a simple harmonic oscillator effected (i.e. driven) by an input voltage source which electromechanically causes the observation of the oscillation, i.e. periodical, electron phenomenon in the Davisson Germer Experiment. This hypothesizes that the crystal lattice can be mechanically modeled as a crystal oscillator circuit. Fortunately,

crystal oscillator circuit analysis is a well-established practice in the electrical engineering science, and permits electrical circuit mathematical modeling with physical motion causes on the resonant frequency of the circuit and not on the sequencing of time:

32 Gehrenbeck, Richard K. 1978. “Electron Diffraction. Fifty Years Ago.” Physics Today p41.

33 Bragg William Henry and Bragg William Lawrence. 1913. The reflection of X-rays by crystals (p431). Proc. R. Soc. Lond. A88 428-438http://doi.org/10.1098/rspa.1913.0040

34 https://pdfserv.maximintegrated.com/en/an/TUT5265.pdf 


$$
\begin{aligned}
& m \ddot{y}+m \gamma \dot{y}+m w_{0} y=F d r i v i n g \\
& w_{0}={\sqrt{k / m_{(3)}^{35}}}
\end{aligned}
$$

where $w 0=\sqrt{ }(k / m)$ is the natural (resonant) frequency of the oscillation derived from the undriven, undamped, simple harmonic oscillator mechanical model: $d^{2} y / d t^{2}=-k / m^{*} y$.

Indeed, the Lorentz. Harmonic Oscillator is a well-established simple harmonic motion linear differential equation system which approximates classically mechanical resonant frequencies, i.e. bodies with masses, as an effect of dipole radiation fields. ${ }^{36}$

The Lorentz Oscillator Model of a damped driven harmonic oscillation is as follows:

$$
\begin{aligned}
& \text { Oscillator Resonance }
\end{aligned}
$$

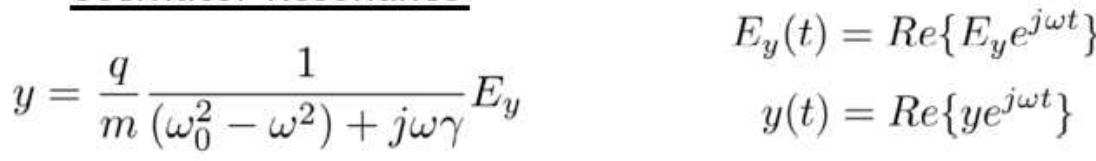

$$
\begin{aligned}
& \text { Figure H Harmonic Oscillator Mode }{ }^{\beta 7}
\end{aligned}
$$

As such, a deeper understanding of the electromagnetic effects on the elastic collision of the electron-crystal phenomena experienced in the Davisson-Germer experiments can demonstrate a more mathematically complete observation of the energy transfer in all physical systems of observation. And this hypothesis interprets the experimental phenomenon of wave constructive interference as the effect of dielectric oscillations. This implies the phenomenon is an effect of electromechanical resonant-driven simple harmonic motion and is therefore the observation of fundamental linear time-invariant motion of

\footnotetext{
35 The Lorentz. Oscillator and its Applications (https:/ / ocw.mit.edu/courses/electrical-engineering-and-computer-science/6007-electromagnetic-energy-from-motors-to-lasers-spring-2011/readings/MIT6_007S11_lorentz.pdf)

36 Electronic Oscillations and Light Scattering. (https://www.vanderbilt.edu/AnS/physics/courses/phys221/Lectures/Lecture27.pdf)

37 The Lorentz Oscillator and its Applications. (https://ocw.mit.edu/courses/electrical-engineering-and-computerscience/6-007-electromagnetic-energy-from-motors-to-lasers-spring-2011/readings/MIT6_007S11_lorentz.pdf)
} 
electromagnetism in time. Indeed, Debye's research further led him to consider the polarization dynamics of the molecules independent of time, i.e. frequency dependent.

\begin{abstract}
"The concept of the orientation of dipolar molecules in an electric field, this time an alternating one, was applied by Debye (1913) in the explanation of the behavior of the two dielectric constants, real and imaginary, that are to be observed. (Permittivity and loss factor are better terms when frequency dependence is involved.) The basic principle is that when the field is applied, or released, a finite time will be required for the molecules to come to their equilibrium orientation because there is a viscous resistance to these rotatory motions. The range of frequency over which the real dielectric constant is variable extends from the static field to one that oscillates so rapidly as not to provide for any rotational motion of the polar molecule at all; the theory thus describes a typical molecular relaxation process." 38 (emphasis added)
\end{abstract}

Thus, the crystal lattice atomic polarity can be theorized as behaving analogously to a Lorentz Harmonic Oscillator piezoelectric material in the presence of an electro-mechanical disturbance, as a classical mechanical model of the electron and X-ray collisions (i.e. corpuscular theory) can.

As such, there is the introduction of electromagnetic theory to classical forces in piezoelectric phenomena providing the bridge between the classical and the quantum in helping to interpret the experimental observations of Davisson and Germer and explain the successful advances of the electrical engineering sciences, e.g. the Fast-Fourier Transform. Namely, the intensity diagrams demonstrate the quantum collisions are dependent on the natural frequency, i.e. resonance, caused by a driven electromagnetic force (the X-Ray or electron). It is precisely at the juncture of electromagnetic polarity where the dynamics can be electro-mechanically equated and diagrammed using electrical circuit equivalence.

We can comparatively mathematically characterize the oscillation effects within an electromagnetic, i.e. frequency-dominant ergo time-invariant, model. In this manner, we can ascertain predictability in the observation of harmonic patterns with the theoretical electro-mechanically induced resonant frequency of the crystals, including the existence of overtone frequencies to

\footnotetext{
${ }^{38}$ Williams, J. (1975). Peter Josseph Wilhelm Debye (p35). Washington, DC: National Academy of Sciences. doi:http://www.nasonline.org/publications/biographical-memoirs/memoir-pdfs/debye-peter.pdf
} 
explain the multiplicity of special reflection angles. Hypothetical resonant frequency effects from the Lorentz Harmonic Oscillator approximations will indicate the presence of dipole moments during photon (e.g. X-ray) and electron crystal elastic collisions. And it further suggests linear time-invariant radiation transfer, i.e. frequency-domain causality, universally in all physical phenomena.

\section{Electromechanical Equivalent Circuit}

A traditional Piezoelectric crystal oscillator circuit model is diagrammed below:

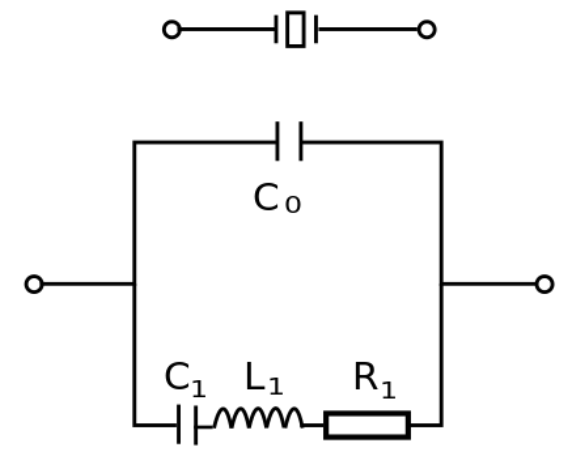

Figure I Crystal Oscillator RLC Circuit Analogue

$$
\begin{aligned}
& Z(s)=\frac{s^{2}+s \frac{R_{1}}{L_{1}}+\omega_{\mathrm{s}}{ }^{2}}{\left(s \cdot C_{0}\right)\left[s^{2}+s \frac{R_{1}}{L_{1}}+\omega_{\mathrm{p}}^{2}\right]} \\
& \Rightarrow \omega_{\mathrm{s}}=\frac{1}{\sqrt{L_{1} \cdot C_{1}}}, \quad \omega_{\mathrm{p}}=\sqrt{\frac{C_{1}+C_{0}}{L_{1} \cdot C_{1} \cdot C_{0}}}=\omega_{s} \sqrt{1+\frac{C_{1}}{C_{0}}} \approx \omega_{s}\left(1+\frac{C_{1}}{2 C_{0}}\right) \quad\left(C_{0} \gg C_{1}\right)
\end{aligned}
$$

Here, we have diagramed an internal resonator (RLC) circuit which will oscillate when the impedance of the crystal's internal induction and its internal capacitance are near identical. The use of nickel, such as in the Davisson-Germer experiment, has been shown, for instance, to be ferromagnetic and thus capable of induction. ${ }^{40}$ Thus, the observation of electron wave harmonics in the "matter-wave" experiment is theoretically proposed to be caused by piezoelectric effects of

\footnotetext{
${ }^{39}$ https://en.wikipedia.org/wiki/Crystal oscillator

${ }^{40}$ https://terpconnect.umd.edu/ wbreslyn/magnets/is-nickel-magnetic.html
} 
electromotive forces (emf) causing the crystal to oscillate (resonant) frequencies in its electrical feedback network, initialized by the electron beam elastic collision (represented by a parallel capacitance component) upon the nickel metal surface. This oscillation necessitates feedback of the DC input voltage to cause the observed harmonics, i.e. amplitude gain, from the beam collector. Indeed, frequency-dependent or time-invariant, mechanics in the electrical engineering domain is very common in analog filters of electromagnetic energy transfer. This system is linear and can be deduced firstly through its electromechanical harmonic oscillator circuit analogy.

Not considered in this approximation are the actual geometrical deformations by the electrons - and by extension photons - upon the crystal lattice. Such considerations, e.g. with finite elemental analysis, can provide a more mathematically rigorous approximation by informing the analysis with more precise frequency-dependent experimental parameters. Neither is there a consideration of the appropriate electrical resistance by the ionization plate (i.e. collector beam intensity measurement). Regardless, it is permissible to understand the changes in beam intensity patterns as pure mathematical demonstrations of electrical feedback amplitude gain or lossless, i.e. perfectly elastic, radiation transfer. This judgment supposes any electromagnetic particle can be completely diagrammed as a complex exponential signal in time, with its continuous-time real frequency components representing electrical motion and its imaginary components representing magnetic motion. Thus, piezoelectric oscillations, such as in the Davisson-Germer experiment, can be theorized to inductively conduct mathematically perfect electric gain through a linear-time invariant signal operation at the moment of collision. 


\section{Mechanical Proof of Electromagnetic Induction Gain}

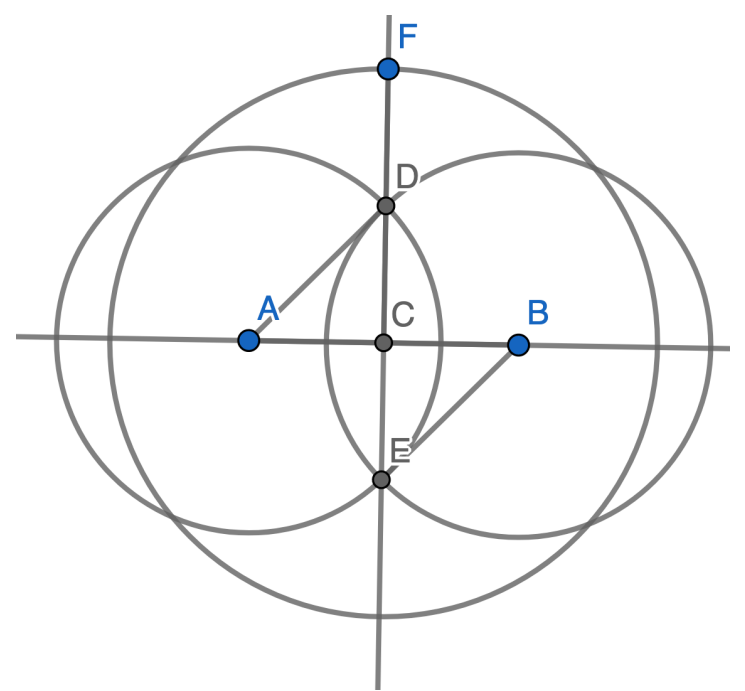

Figure J Mechanical Construction of electromagnetic radiation induction caused by perfectly elastic (kinetic) electron collisions forming a linear superposition of electrical feedback at Point $C$. Electrons are diagrammed as complex exponential units of motion in space which presuppose the measure of amplitude as a self-certain geometrical relation in all quanta capable of mathematical scientific inspection.

Consider an electron body at point A moving rectilinearly in time. This body can be diagrammed with quantities of motion independent of time. Specifically, the electron can be diagrammed completely with the complex exponential, where the electrical and magnetic sinusoidal waveform components are self-contained, i.e. completely bounded, in the analytically geometrical number corresponding to electrical and magnetic motion superimposed at any point A in space. And since the natural mechanics of the complex exponential is simple harmonic motion in the complex domain, we have an ideal mathematical correspondence of a stable unit of electromagnetic radiation propagation empirically measurable in volts, wherein the electrical body can be simultaneously diagrammed as uniform motion (a) rectilinearly as a Galilean body with mass, or (b) circularly as the pure mathematical representation of motion as an absolute quantitative measure of the radiation's amplitude (magnitude), by mathematical induction in the geometrical analysis of the observation. This induction is achieved through the mathematically perfect transformation of the rectangular 
algebraic relation of the electron position into polar coordination form of the position, thereby demonstrating the self-certain quantity of motion, i.e. information, of radiation inspected: ${ }^{41}$

$z=a+j b=r \cos w+j r \sin w=r e^{j w}|z|=\operatorname{sqrt}\left(a^{2}+b^{2}\right)=r z=|z| e^{j w}$

Next consider its collision with another electrical body extending with center B at a selfcertain occurrence diagrammed at midpoint $\mathrm{C}$. This collision can be found, for instance, in a piezoelectric oscillation event, and diagrammed as a linear time-invariant operator. Our analysis can be informed deeper with the understanding of the complex frequency number, a necessarily periodical and numerically complex self-certain agreement between the position of the point moving uniformly on the circle and its center, ${ }^{42}$ to inspect the uniform motion of the mass of the electrical body to be mechanically rotating around a fixed center, circularly moving invariantly to time, with known magnitude as a purely numerical measure of its quantity of motion in volts. It is through this understanding where we can mathematically completely diagram the collision at point $\mathrm{C}$ as an electromagnetic radiation construction phenomenon using complex conjugate summation to determine the amplitude extension of the phase-shift oscillation as the length CF:

$$
\begin{aligned}
& A=e^{j * w} ; A^{*}=e^{-j * w} ; B=A^{*} ; A+B=(\cos w+j * \sin w)+(\cos w-j * \\
& \sin w)=2 \cos w=A B ; A B=C F
\end{aligned}
$$

In other words, it is claimed electron elastic collisions, which can be applied to the DavissonGermer experimental results, processes imaginary quantities of magnetic periodical motion summated in opposite rotations - specifically $\pi$ radians out of phase - into real experimental amplifications of electrical measurements. This graphical diagram of the linear superposition of time-invariant quantities of electromagnetic radiation at Point $C$ visually demonstrates mathematically scientific

\footnotetext{
${ }^{41}$ Lathi, B. (2005). Linear Systems and Signals (2nd ed.) (pg. 6). New York, NY: Oxford University Press.

42 See: Hendrik W. Bode Network Analysis Feedback Amplifier Design (1945).
} 
amplitude gain caused by electromagnetic induction as the empirical phenomenon of extending imaginary quantities of magnetic energy into real electrical quantities measurable in the continuous-time domain. This measurement can be ideally diagrammed as a phasor in a polar coordination system with a complex exponential evaluated at the special reflectance angles in the crystal experiments. It is conjectured this is the principle phenomenon of all radiation transfer in electrical feedback circuits and indeed in all electromagnetic constructive interference. This is most especially relevant in biological tissue which is principally piezoelectric in nature.

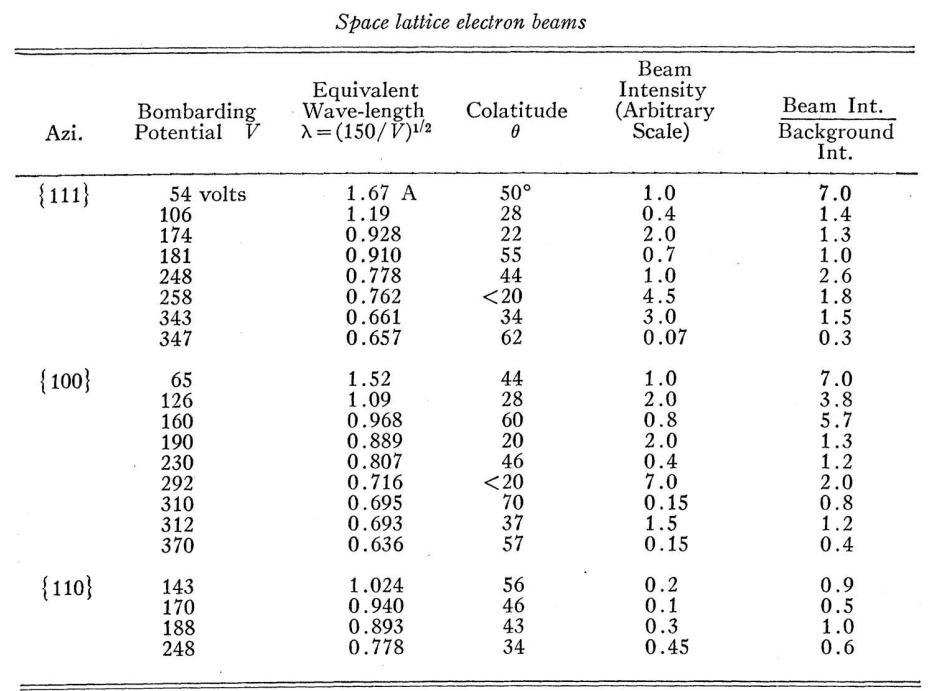

In considering the conditions under which such beams will occur it will be convenient to regard the crystal as built up of $\{111\}$-planes of atoms parallel to the principal facets, and to picture the radiation scattered by the crystal as made up of the contributions from all such planes. This viewpoint has a distinct advantage, in the present case, over regarding diffraction beams as built up of contributions regularly reflected from the Bragg atom-planes. The amplitude of the radiation proceeding in a given direction from the crystal is then to be regarded as the sum of the amplitudes (with due regard to phase) of the increments of radiation proceeding in the same direction from all such $\{111\}$-planes of atoms-or more precisely, the fractions of such increments that actually escape from the crystal.

If we imagine a system of Cartesian coordinates with its origin at the center of a surface atom, its positive $z$-axis extending outward from the facet, and its positive $x$-axis lying in one of the $\{110\}$-azimuths of the crystal, Figure $K \sim .85 d \dot{B}$ peak, gain in electromagnetically induced superposition in the Davisson-Germer Experimental Results ${ }^{43}$

Henceforth, it is claimed that the Davisson-Germer experiment demonstrates sufficient reason to mechanically diagram all electromagnetic phenomena as linear-time invariant causal

\footnotetext{
${ }^{43}$ Davisson, C. and Germer, L.H. (1927) Diffraction of Electrons by a Crystal of Nickel. Physical Review, 30, 705-740. http://dx.doi.org/10.1103/PhysRev.30.705
} 
operations, contrary to the experimenters judgments of the electron phenomenon being optical. Such electromagnetic phenomena include biological physical matter which has been extensively demonstrated to be piezoelectric and electroconductive. including DNA, amino acids, etc. ${ }^{44}$ This empirical evidence justifies inspecting cellular biological organisms as stable electromagnetic radiation transmission systems, with their continuous-time adaptations mathematically formalized as feedback control responses. It follows that there is a correspondence between feedback order and cellular biological fitness or the quantitatively measurable ability for the cellular organism to replicate its BIBO stable radiation propagation in time.

\footnotetext{
${ }^{44}$ Shamo MH, Lavine LS. Piezoelectricity as a fundamental property of biological tissues. Nature. 1967;213:267-269.
} https://doi.org/10.1038/213267a0 
Figures

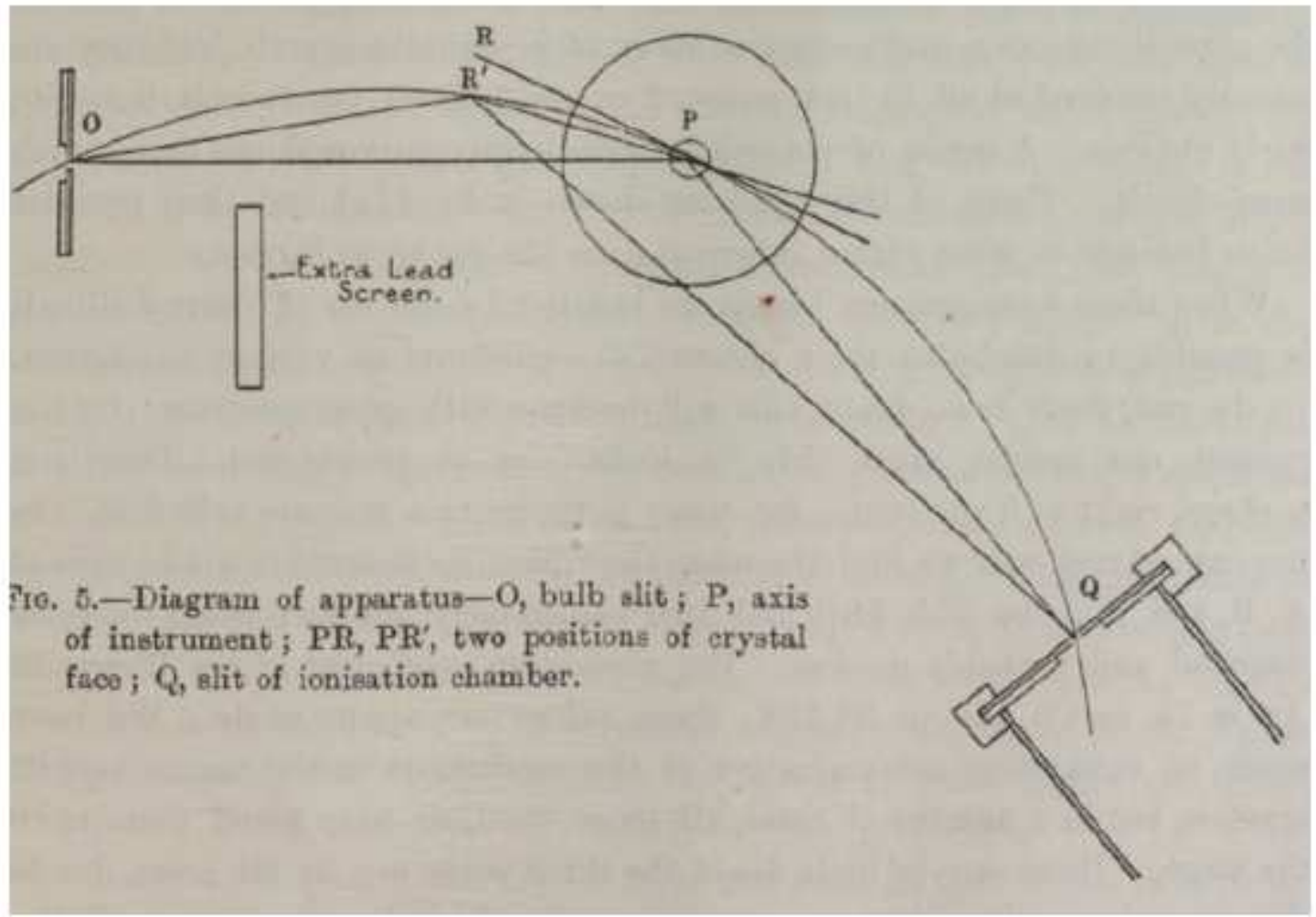

\section{Figure 1}

Figure A Bragg Experimental Setup with X-ray emitting from a point source $\mathrm{O}$ and reflecting at a "special angle".

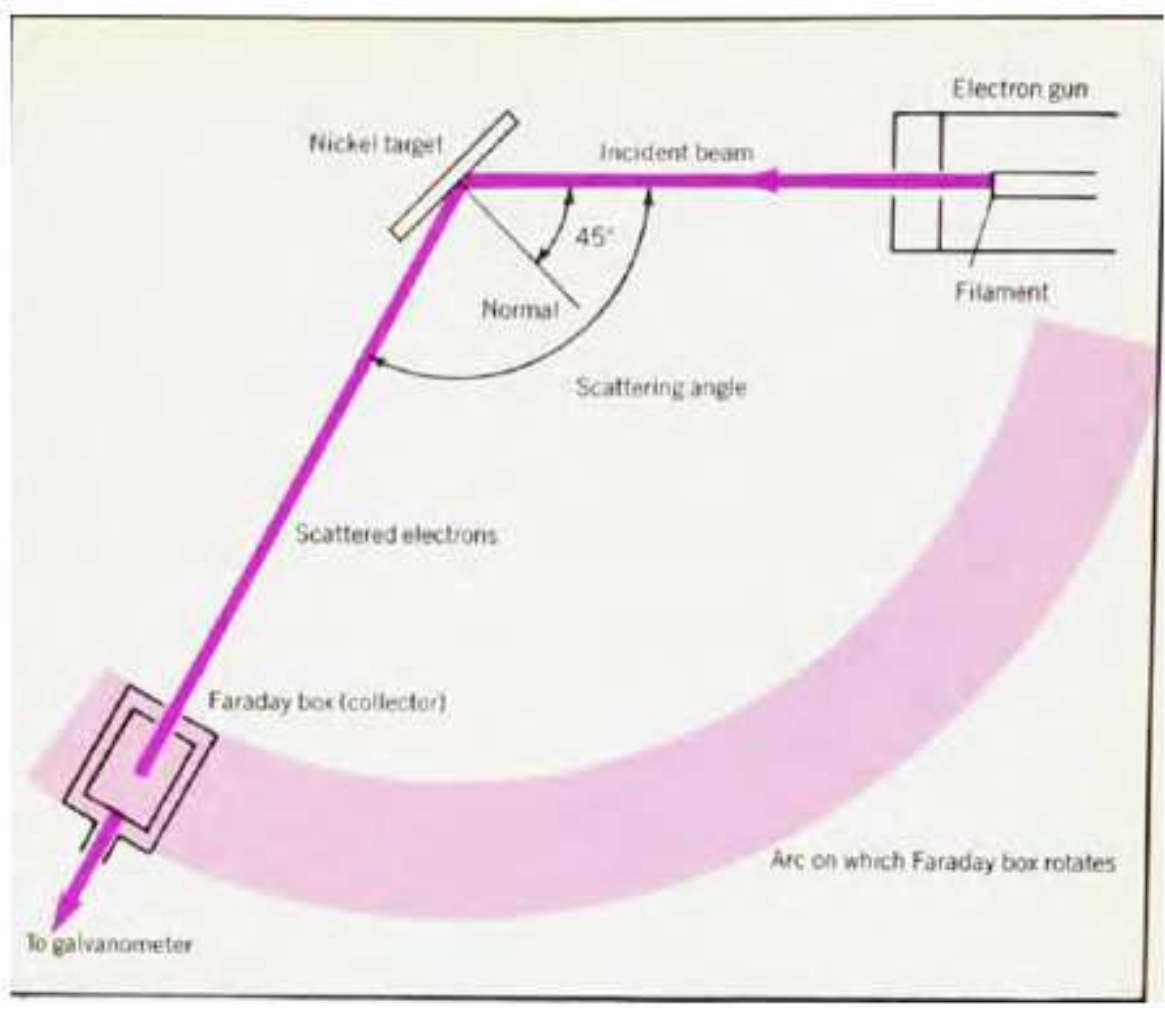


Figure 2

Figure B Davisson-Germer Experimental Setup
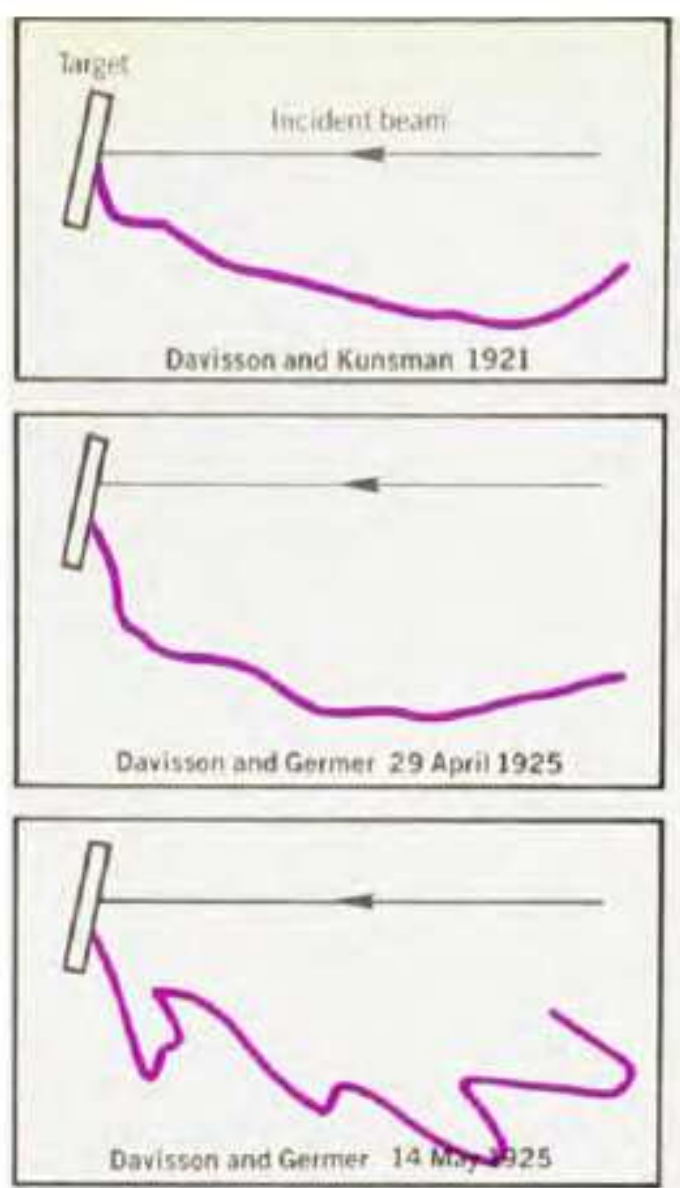

Figure 3

Figure C Electron Diffraction Experimental Results 

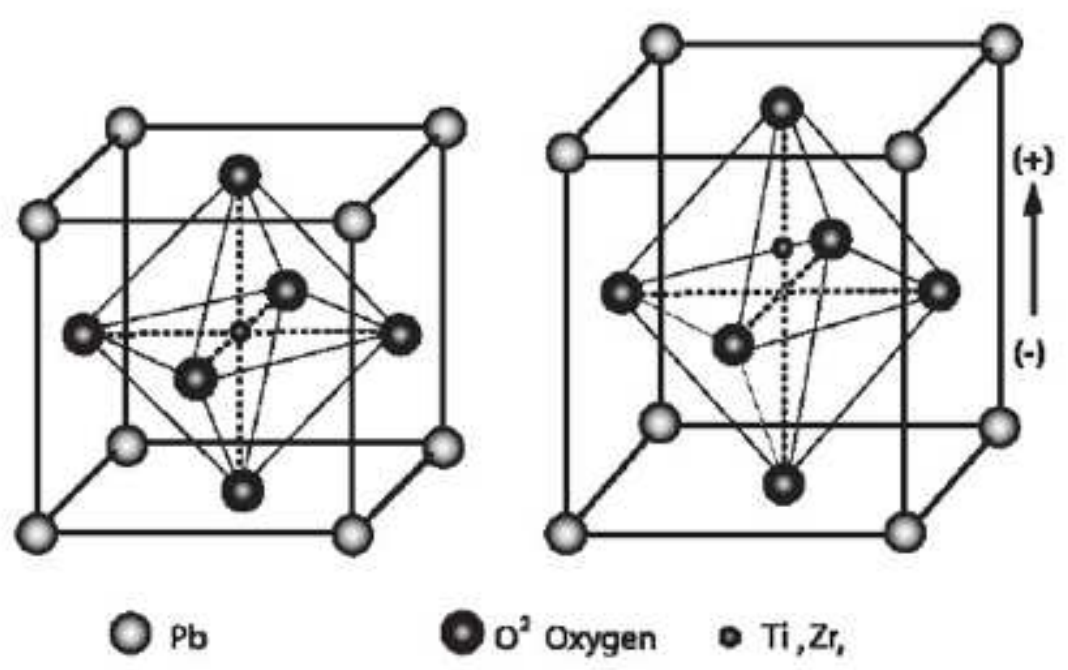

Figure 2.1. Crystalline structure of a piezoelectric ceramic, before and after polarization

\section{Figure 4}

Figure D Piezoelectric effect of Crystal acted on by an electromagnetic "force"

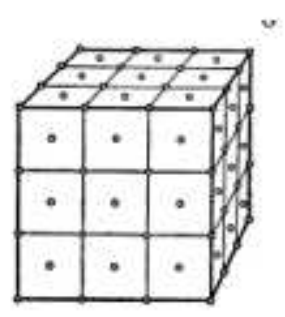

(a)

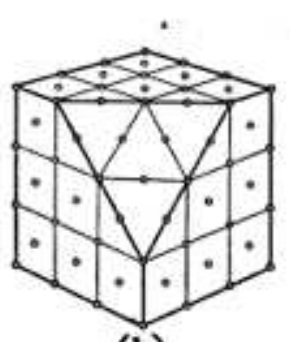

(b)

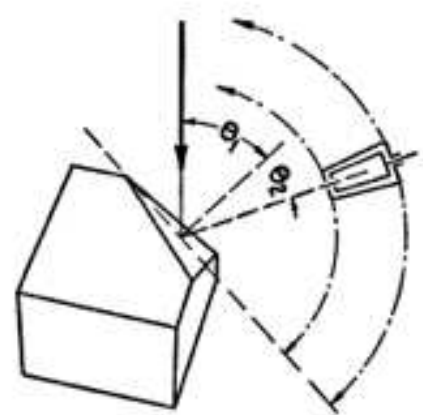

(c)

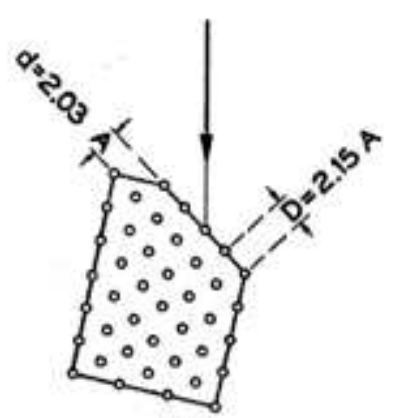

(d)

FIOURE 1

Schematic diagrams indicating the experimental arrangement for measuring the reflection of electrons.

Figure 5

Fig E Davisson-Germer nickel crystal suggesting piezoelectric occurrence 


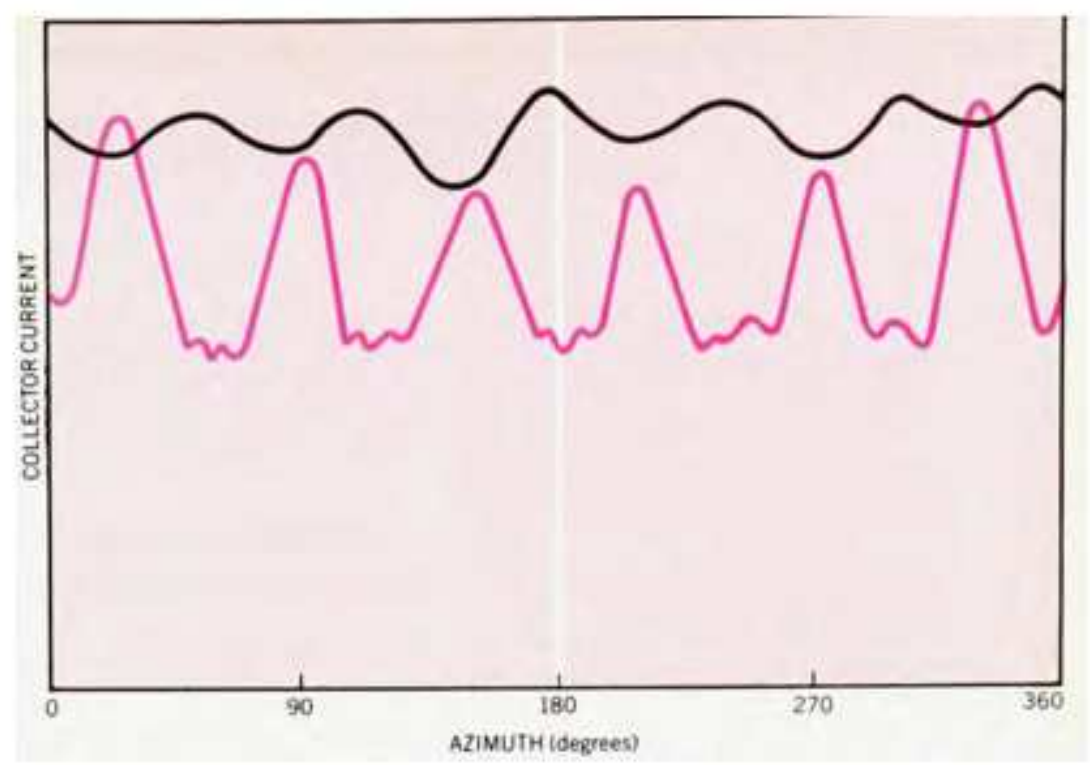

Figure 6

Figure F Davisson-Germer Scattering Results Suggesting Amplifier Gains of Piezoelectric Oscillation Effects

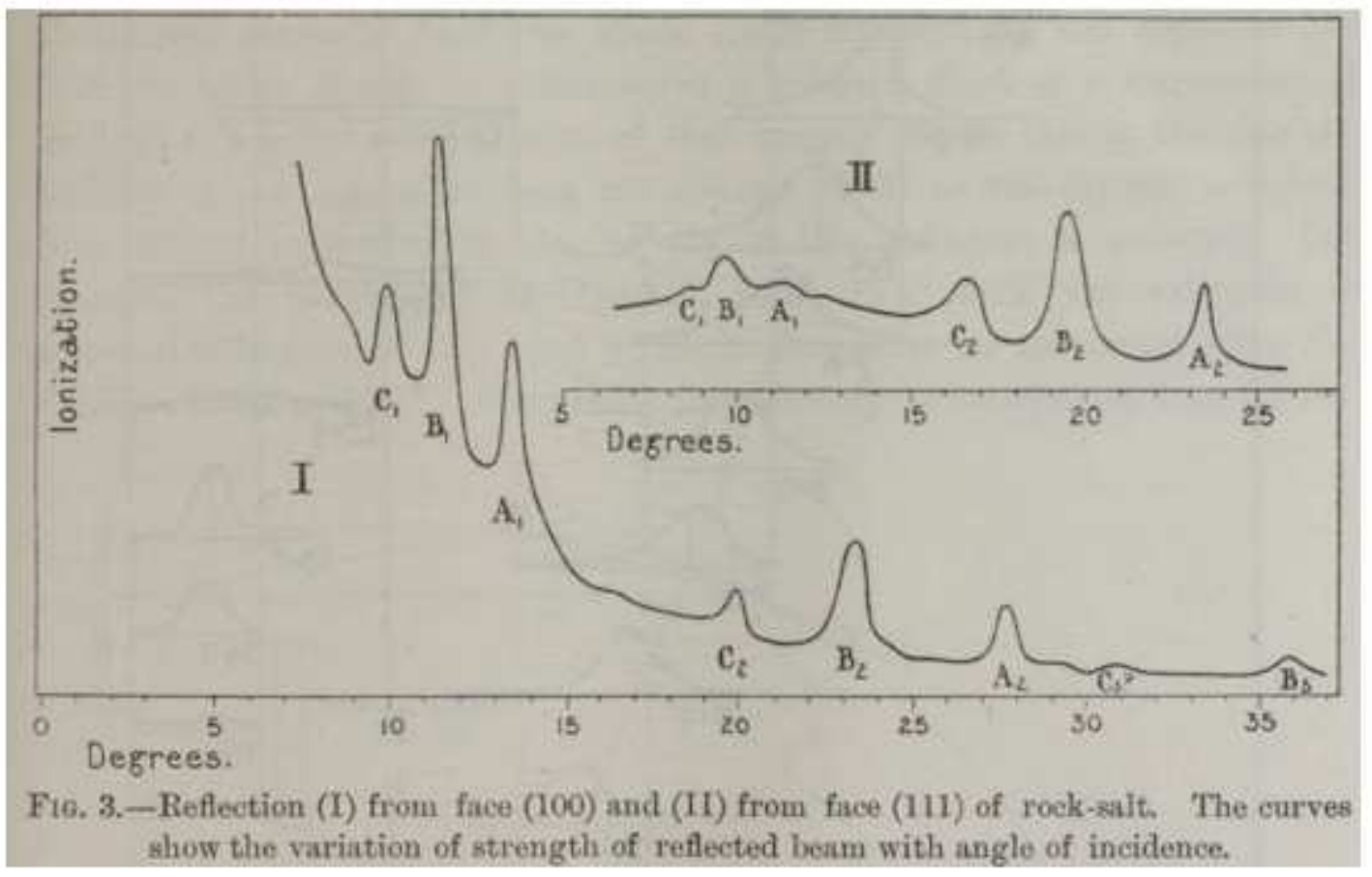

Figure 7

Figure G Bragg Scattering Angles Suggesting Overtone Frequencies of Piezoelectric Oscillation Effects of X-Ray Energy 


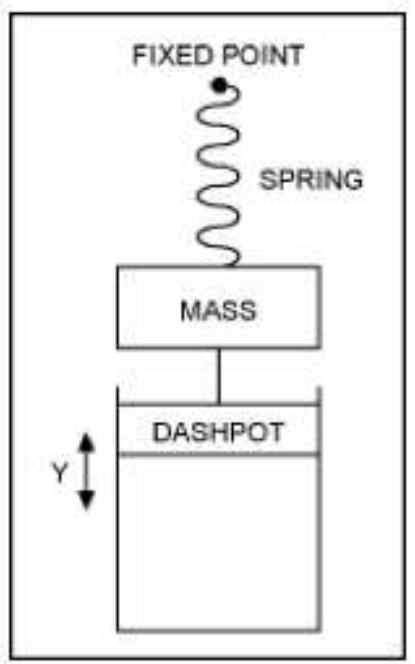

Figure 8

Figure H Damped Harmonic Oscillator Mechanical Model
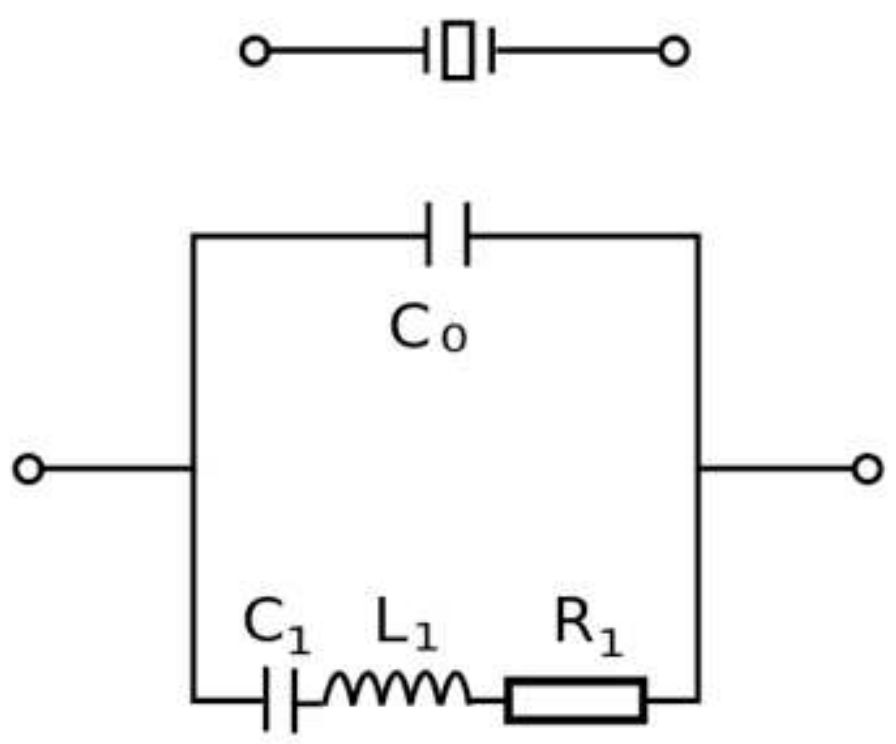

Figure 9

Figure I Crystal Oscillator RLC Circuit Analogue 


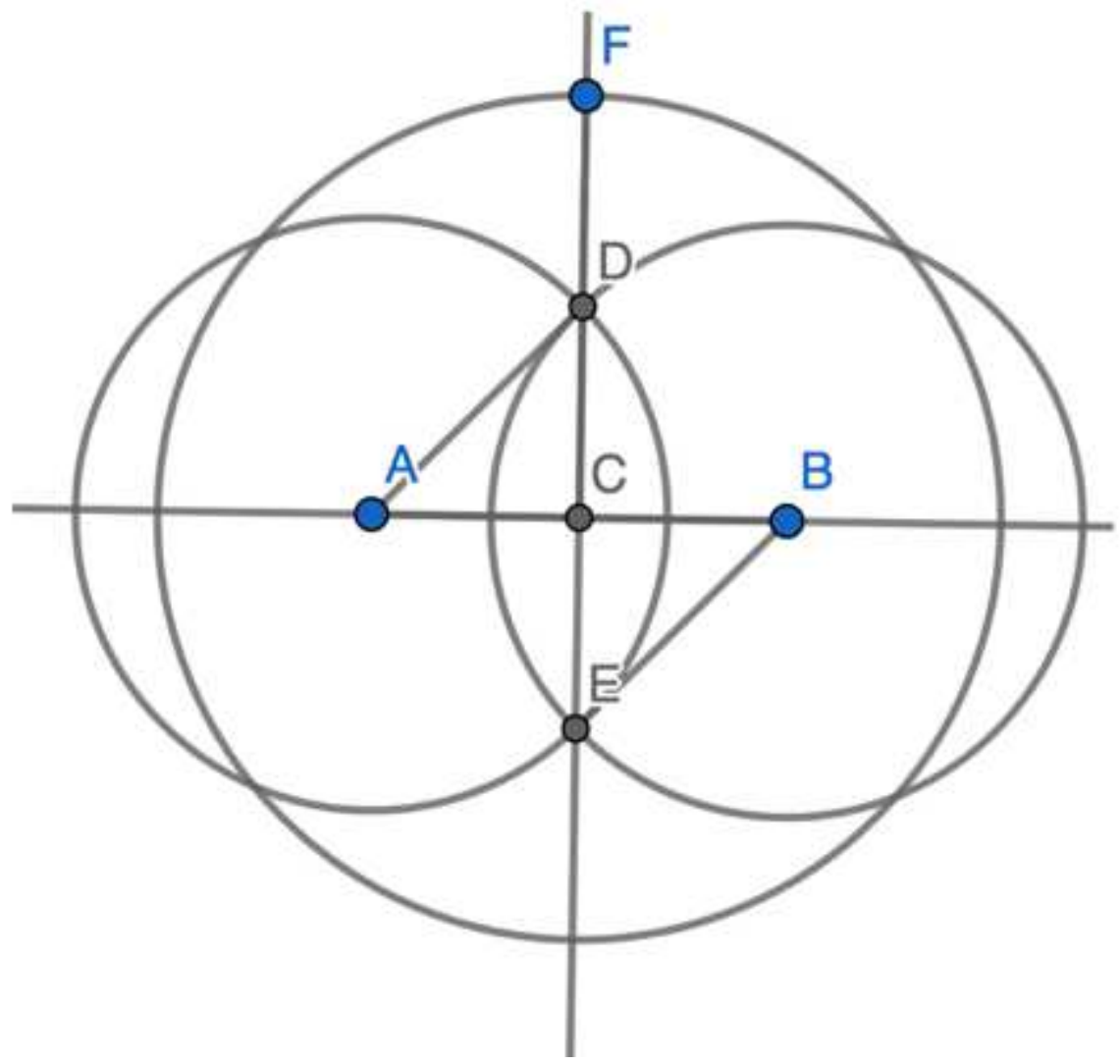

Figure 10

Figure $\mathrm{J}$ Mechanical Construction of electromagnetic radiation induction caused by perfectly elastic (kinetic) electron collisions forming a linear superposition of electrical feedback at Point C. Electrons are diagrammed as complex exponential units of motion in space which presuppose the measure of amplitude as a self-certain geometrical relation in all quanta capable of mathematical scientific inspection. 
Space lattice electron beams

\begin{tabular}{|c|c|c|c|c|c|}
\hline Azi. & $\begin{array}{l}\text { Bombarding } \\
\text { Potential V }\end{array}$ & $\begin{array}{c}\text { Equivalent } \\
\text { Wave-length } \\
\lambda=(150 / V)^{1 / 2}\end{array}$ & $\underset{\theta}{\text { Colatitude }}$ & $\begin{array}{c}\text { Beam } \\
\text { Intensity } \\
\text { (Arbitrary } \\
\text { Scale) }\end{array}$ & $\frac{\text { Beam Int. }}{\begin{array}{c}\text { Background } \\
\text { Int. }\end{array}}$ \\
\hline$\{111\}$ & $\begin{array}{l}54 \text { volts } \\
106 \\
174 \\
181 \\
248 \\
258 \\
343 \\
347\end{array}$ & $\begin{array}{l}1.67 \mathrm{~A} \\
1.19 \\
0.928 \\
0.910 \\
0.778 \\
0.762 \\
0.661 \\
0.657\end{array}$ & $\begin{array}{r}50^{\circ} \\
28 \\
22 \\
55 \\
44 \\
<20 \\
34 \\
62\end{array}$ & $\begin{array}{l}1.0 \\
0.4 \\
2.0 \\
0.7 \\
1.0 \\
4.5 \\
3.0 \\
0.07\end{array}$ & $\begin{array}{l}7.0 \\
1.4 \\
1.3 \\
1.0 \\
2.6 \\
1.8 \\
1.5 \\
0.3\end{array}$ \\
\hline$\{100\}$ & $\begin{array}{r}65 \\
126 \\
160 \\
190 \\
230 \\
292 \\
310 \\
312 \\
370\end{array}$ & $\begin{array}{l}1.52 \\
1.09 \\
0.968 \\
0.889 \\
0.807 \\
0.716 \\
0.695 \\
0.693 \\
0.636\end{array}$ & $\begin{array}{r}44 \\
28 \\
60 \\
20 \\
46 \\
<20 \\
70 \\
37 \\
57\end{array}$ & $\begin{array}{l}1.0 \\
2.0 \\
0.8 \\
2.0 \\
0.4 \\
7.0 \\
0.15 \\
1.5 \\
0.15\end{array}$ & $\begin{array}{l}7.0 \\
3.8 \\
5.7 \\
1.3 \\
1.2 \\
2.0 \\
0.8 \\
1.2 \\
0.4\end{array}$ \\
\hline$\{110\}$ & $\begin{array}{l}143 \\
170 \\
188 \\
248\end{array}$ & $\begin{array}{l}1.024 \\
0.940 \\
0.893 \\
0.778\end{array}$ & $\begin{array}{l}56 \\
46 \\
43 \\
34\end{array}$ & $\begin{array}{l}0.2 \\
0.1 \\
0.3 \\
0.45\end{array}$ & $\begin{array}{l}0.9 \\
0.5 \\
1.0 \\
0.6\end{array}$ \\
\hline
\end{tabular}

In considering the conditions under which such beams will occur it will be convenient to regard the crystal as built up of $\{111\}$-planes of atoms parallel to the principal facets, and to picture the radiation scattered by the crystal as made up of the contributions from all such planes. This viewpoint has a distinct advantage, in the present case, over regarding diffraction beams as built up of contributions regularly reflected from the Bragg atom-planes. The amplitude of the radiation proceeding in a given direction from the crystal is then to be regarded as the sum of the amplitudes (with due regard to phase) of the increments of radiation proceeding in the same direction from all such $\{111\}$-planes of atoms-or more precisely, the fractions of such increments that actually escape from the crystal.

If we imagine a system of Cartesian coordinates with its origin at the center of a surface atom, its positive $z$-axis extending outward from the facet, and its positive $x$-axis lying in one of the $\{110\}$-azimuths of the crystal,

than ntam antare noris at the nointe

\section{Figure 11}

Figure $\mathrm{K} \sim .85 \mathrm{~dB}$ peak gain in electromagnetically induced superposition in the Davisson-Germer Experimental Results 